\title{
LARYNGOMALACIA: DIAGNOSIS AND MANAGEMENT
}

\author{
Z. Mandour, H.M. Abdel Fattah, A.E.H. Gaafar \\ Otorhinolaryngology, Alexandria University, Alexandria, Egypt
}

Background: Laryngomalacia is the most common cause of congenital stridor. It usually presents by high pitched inspiratory stridor which is often present at birth and is usually noticed by 2 weeks of age.

Aim: The aim of this study was to assess clinical presentation, management and prognosis of infants and children suffering from laryngomalacia presented to our department in the period of 5 years.

Methods: Retrospective analysis of the medical sheath records of newborns and infants suffering of laryngomalacia were reviewed regarding demographic data, clinical presentation, diagnosis and management.

Results: Fifty eight infant and child were included in the study. They were 33 males (57\%) and 25 females (43\%). Their age at presentation ranged from 2 - 13 months. Diagnosis was done using laryngoscopy under general anesthesia with spontaneous breathing in 49 patients $(85 \%)$ and by using flexible nasopharyngolaryngoscopy under topical anesthesia in 9 patients (15\%). Conservative treatment was given for all cases in the form of diet modification, lansoprazole and Domperidone. For mild cases, gradual improvement occurred within 1 to 3 months. For severe cases, surgical intervention was planned. Indications for surgical intervention were severe airway obstruction with attacks of cyanosis, feeding difficulties and aspiration, weight loss and failure to thrive.

Conclusion: Laryngomalacia is the most common congenital anomaly of the larynx. It usually presents within 2 weeks after birth. Diagnosis depends on visualization of the larynx during respiration. Conservative treatment is the rule. Surgical treatment is only indicated in $10 \%$ of cases 